\title{
Identification of breast cancer using analysis of thermal signals by nonlinear dynamics methods
}

\author{
by E.Gerasimova*, O. Plekhov *, Yu.Bayandin*, O.Naimark*, G.Freynd**
}

* Institute of Continuous Media Mechanics of the Ural Branch of the Russian Academy of Sciences, 1 , Ak. Korolev street, 614013, Perm, Russia, egerasimova@icmm.ru

** Perm State Medical Academy, 26, Petropavlovskaya street, 614990, Perm, Russia, kpa.pgma@mail.ru

\section{Introduction}

At present, infra-red thermography is a promising non-invasive tool for functional diagnosis as it is able to facilitate data visualization and to provide large amounts of data. It has found wide application in different areas of medicine, including breast oncology. Interpretation of infrared imaging data is carried out using the Marseilles Analytic System [1]. Thermal features are divided into Signs and Criteria based on their established characterization of breast disease.

This paper presents the analysis of temperature dynamics by nonlinear dynamics methods. It is supposed that with this approach the significant informative characteristics of the heat pattern of the breast surface can be obtained. Determination of new classification parameters will allow early recognition of breast surface thermographic abnormalities not expressed clearly due to various factors, such as when small tumor located in a deep region, or environmental influences that make breast disease difficult to find. The results of this study might essentially improve the information potential of infrared thermography in medicine.

\section{Results}

Dynamic infrared imaging of breasts was undertaken preoperatively in 46 cases with histopathologically proven benign and malignant breast tumors. Using the Fourier analysis, wavelet transformation and phase diagrams, we studied the temperature signals from the normal and tumor regions (figure 1). It has been found that there are differences between the obtained spectra and the phase diagrams for normal tissue and a tumor (figure 2).

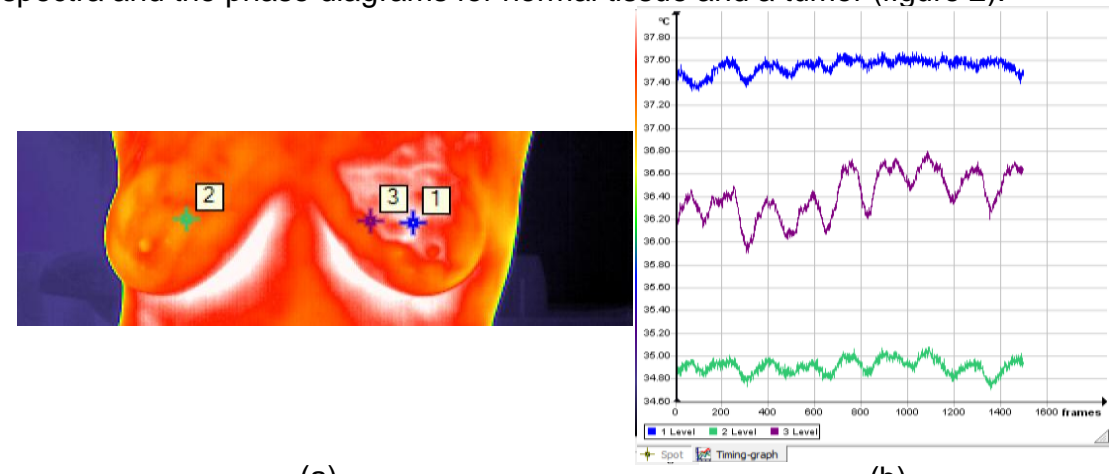

(a)

(b)

Figure 1: Breast thermogram (a) with the investigated thermal signals (b)

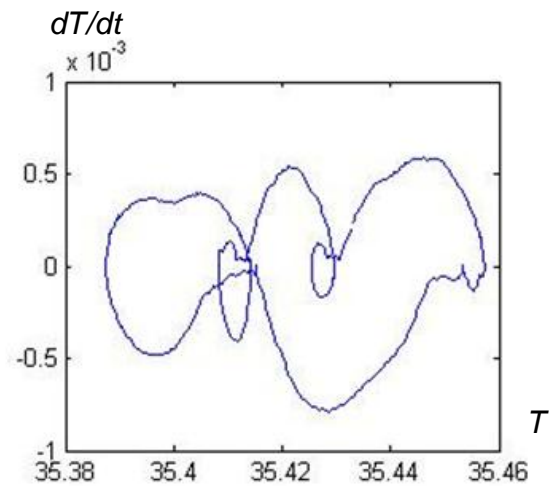

(a)

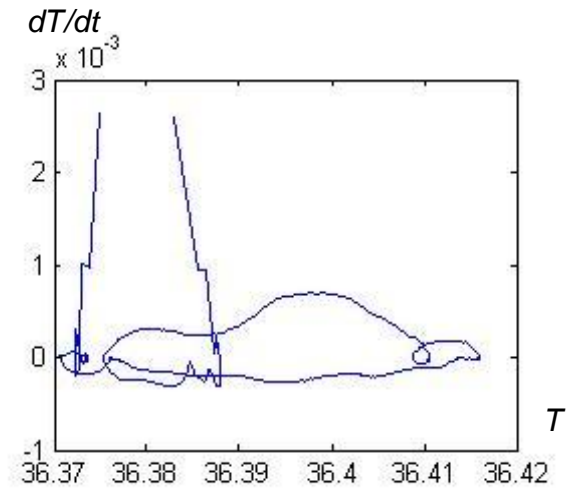

(b)

Figure 2: Signal phase diagrams for the normal tissue (a) and the tumor (b) 
It is shown that the chaotic phase diagram corresponds to a healthy tissue, while for cancerous tissues the irregular shapes in the phase space are typical. This is indicative of the inability of the abnormal tissue to adapt to the external influence. These results correspond to the health concept that considers the transition from "health" to "disease" as the process of reducing adaptive capabilities of an organism [2].

The temperature characteristics of various sections of the excised breast tissue obtained at the stage of surgical intervention have been analyzed to investigate the correlation properties of temperature signals of tumor tissue, perifocal tissue (adjacent to the tumor) and healthy tissue (figure 3). The results of our investigation have indicated that the temperature signals of healthy and perifocal tissues are anticorrelated, whereas in the tumor the thermal noise correlation has been observed (figure 4).

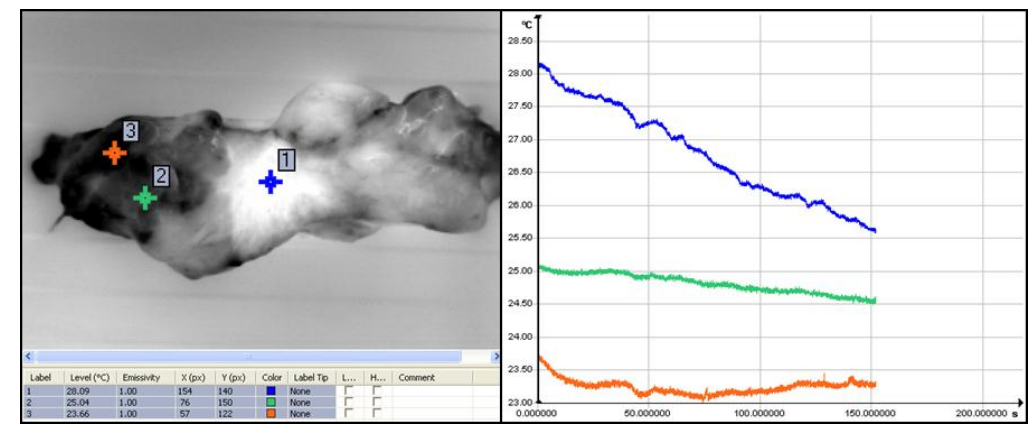

(a)

(b)

Figure 3: The temperature distribution on the surface of a breast tissue section (a) and the corresponding thermal signals (b)

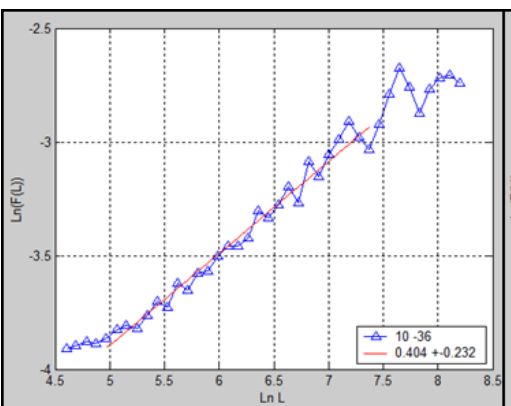

(a)

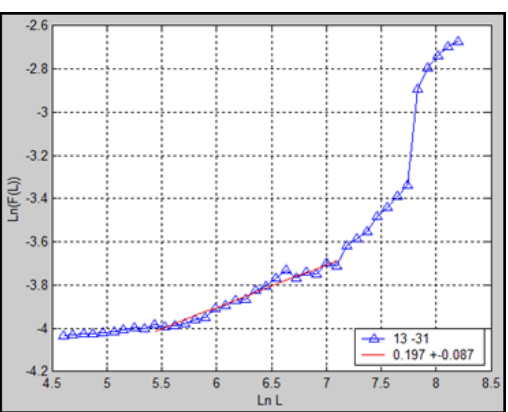

(b)

Figure 4: Correlation properties of thermal signals in a tumor (a) and in healthy tissue (b) (according to Detrended Fluctuation Analysis)

The research was supported by the Russian Foundation for Basic Research (grant № 10-01-96051-r_ural_a).

\section{REFERENCES}

[1] Joseph D . Bronzino, ed., The Biomedical Engineering Handbook, Third Edition: Medical Devices and Systems, Taylor \& Fransic Group, New-York, 2006

[2] R.M. Baevsky, A.P. Berseneva, Assessment of adaptation capacity of an organism and the risk of disease, Medicine, Moscow, 1997. 